\title{
Momento de formación y periodicidad de los microincrementos de crecimiento en otolitos de larvas de pejerrey (Austromenidia regia) mantenidas en laboratorio
}

\author{
Jessica Peñailillo P. y Miguel Araya C. \\ Departamento Ciencias del Mar, Universidad Arturo Prat \\ Casilla 121, Iquique, Chile
}

RESUMEN. Larvas de pejerrey (Austromenidia regia) se mantuvieron en laboratorio, por un período de 26 días, con el objeto de conocer la periodicidad y el momento de formación del primer microincremento de crecimiento en el otolito sagitta. La temperatura y el fotoperíodo se mantuvieron constantes. Las larvas se alimentaron ad libitum con plancton, alimento artificial y nauplios de Artemia sp. Se sacrificaron 5 larvas a intervalos regulares, a las que se les midió la longitud estándar y extrajeron los otolitos sagittae. Los otolitos fueron leídos por dos lectores, dos veces cada uno; las lecturas con más de tres microincrementos de diferencia se descartaron.

Al eclosionar las larvas presentan una longitud estándar promedio de 7,3 mm, alcanzando al final del período experimental un promedio de 12,3 $\mathrm{mm}$. El análisis de regresión entre el número de microincrementos y la edad en días entrega los siguientes parámetros: $\mathrm{a}=5,86 \mathrm{y} \mathrm{b}=0,98$, resultando la pendiente significativamente igual a uno. El intercepto de la regresión indica que las larvas eclosionan con aproximadamente 6 microincrementos, lo cual se corroboró con el análisis de otolitos de larvas recién eclosionadas.

Palabras clave: otolito, microincremento, Austromenidia regia, validación, pejerrey.

\section{Formation moment and periodicity of the growth microincrements in the otoliths of pejerrey larvae (Austromenidia regia) maintained in the laboratory}

\begin{abstract}
In order to know the periodicity and the moment of first growth microincrement formation in the sagitta otolith of pejerrey larvae (Austromenidia regia) were maintained in the laboratory for 26 days. The photoperiod and temperature were maintained constant. Larvae were fed ad libitum with plankton, artificial food for fishes and Artemia sp. nauplii. Five larvae were sacrificed at regular intervals; it standard length was measured then the sagittae and lapillus otoliths were extracted. The otoliths were reading by two readers counting twice each one, with more than three microincrements of diference in the reading were discarded.

At larvae hatch present an average standard length of $7,3 \mathrm{~mm}$, reaching an average standard length of $12,3 \mathrm{~mm}$ at the final of the experimental period. The regression analysis between the number of microincrements and the age in days, delivery the following parameters: $\mathrm{a}=5,86 \mathrm{y} b=0,98$, resulting the slope significantly equal to one. The regression intercept indicates that larvae hatching with approximately 6 microincrements, which it was corroborated with the analysis of otoliths of larvae newly hatching.
\end{abstract}

Key words: Otolith, microincrements, Austromenidia regia, validation, pejerrey.

\section{INTRODUCCION}

La presencia de microincrementos en los otolitos de larvas de peces ha dado apoyo a los estudios en la determinación de la edad, crecimiento, mortalidad e historia de la vida temprana (Moksness, 1992; Panella, 1971, 1980; Methot y Kramer, 1979; Taubert y Coble, 1977; Townsed y Graham, 1981;
Brothers et al., 1976). Estos estudios dependen de los siguientes supuestos: que los microincrementos de crecimiento sean depositados diariamente (Panella, 1971, 1980; Campana y Neilson, 1985; Jones y Brothers, 1987), o con alguna tasa constante (Lough et al., 1982); que la fecha de formación del microincremento inicial sea conocida (Wilson y Larkin, 1980; Volk et al., 1984); y que exista una 
relación funcional entre el crecimiento del otolito y la longitud de la larva (Laroche et al., 1982).

Varios trabajos han demostrado que la estructura del crecimiento en otolitos de peces tiene una base diaria, entre ellos: Barkman (1978) en Menidia menidia; Brothers et al. (1976) en larvas de Engraulis mordax y Leuresthes tenuis, en juveniles de Morone saxatilis, Clevelandia ios, y en adultos de Merluccius angustimanus; Tanaka et al. (1981) en juveniles de Tilapia nilotica; Townsed y Graham (1981) en larvas de Clupea harengus; Castillo et al. (1985) en larvas de Sardinops sagax; Micheli y Huaquín (1985) en larvas de Basilichthys australis; y Jones y Brothers (1987) en larvas de Morone saxatilis.

Por otro lado existen trabajos que indican que la generación de microincrementos en los otolitos no siempre es diaria. Por ejemplo, Geffen (1982) demostró en laboratorio que la tasa de formación de anillos en otolitos de larvas de Clupea harengus y Scopthalamus maximus es menor a un incremento por día y que sería dependiente de la tasa de crecimiento del pez. Además, se tiene la presencia de anillos de crecimiento sub diarios, ej. En Lepomis gobbosus, los cuales se distinguen de los anillos diarios porque los primeros no se encuentran bien definidos (Taubert y Coble, 1977).

Radtke y Dean (1982) señalan que una importante área de investigación en el campo de la edad y el crecimiento, es el estudio experimental de los factores que influyen en la formación de los microincrementos en los otolitos. Así, que al determinar la edad en larvas y juveniles de peces hay que tener en cuenta ciertas variables, como la temperatura (Geffen, 1983; Neilson y Geen, 1985), alimento (Geffen, 1982; Neilson y Geen, 1982) fotoperíodo (Geffen, 1982; Taubert y Coble, 1977; Tanaka et al., 1981), y un ritmo circadiano endógeno (Campana y Neilson, 1985; Mugiya et al., 1981). Estos podrían afectar el mecanismo de formación de los microincrementos, ya sea en el espesor o en el número de líneas, por lo que es conveniente demostrar que los microincrementos tienen una naturaleza diaria antes de determinar la edad en cualquier especie.

Por otra parte, la exactitud en la estimación de la edad en larvas parece estar relacionada con la formación del primer anillo de crecimiento (Campana y Moksness, 1991); es decir, al momento de formación del primer microincremento. En varias especies éste se encuentra asociado al término de la absorción del vitelo (Alshuth, 1988; Brothers et al., 1976; Herrera et al., 1985). En Euthynus pelamis la aparición del primer incremento es un día después de la eclosión o tres días post-fertilización (Radtke, 1984); en Leuresthes tenuis al eclosionar y algunos en la pre-eclosión (Brothers et al., 1976); y en Theragra chalcograma al eclosionar (Nishimura y Yamada, 1984).

Ante lo expuesto y a la necesidad de contar con información de los recursos explotados por el sector artesanal, es que el objetivo del presente estudio es determinar el momento de formación del primer microincremento y la periodicidad de éstos en los otolitos sagittae de larvas de Austromenidia regia mantenidas en condiciones de laboratorio.

Austromenidia es un género con especies marinas que se encuentran distribuidas en Chile, Argentina y Perú, teniendo así a Austromenidia laticlavia y Austromenidia regia como probables sinónimos (Campos, 1984). La nomenclatura utilizada en este estudio es Austromenidia regia, ya que A. laticlavia no ha sido validada como especie.

Austromenidia regia (pejerrey de mar) es un recurso de importancia relativa en la pesquería artesanal en Chile. En los últimos años este ha experimentado variabilidad en sus desembarques, siendo mayores en 1989 y 1990 con 2.327 y 5.532 t respectivamente para luego decaer en 1994 a $771 \mathrm{t}$ (SERNAP, 1994). Por otra parte, en Perú es uno de los recursos que sustentan la pesquería artesanal, en cuyo mercado interno es aceptado para el consumo por el agradable sabor y buena textura de su carne (Véliz e Insil, 1988).

\section{MATERIALES Y METODOS}

Se colectaron huevos de Austromenidia regia en el sector de Pozo Toyo (20²5'S y $\left.70^{\circ} 15^{\prime} \mathrm{W}\right)$, mediante buceo apnea. En el laboratorio fueron separados de acuerdo al estado de desarrollo (Fisher, 1963), y posteriormente puestos en acuarios circulares de 31 de capacidad, a temperatura ambiente. Los acuarios fueron revestidos con papel carbón, para permitir un mejor contraste en la visualización de las presas por parte de las larvas (Blaxter, 1969 fide Herrera y Balbontín, 1983; Lasker et al., 1970).

Las larvas se mantuvieron en agua de mar filtra- 
da y esterelizada con UV, con un $\mathrm{pH}$ de 7,8 $\pm 0,2$ y una temperatura de $17,6 \pm 0,4^{\circ} \mathrm{C}$. El primer día el agua de mar fue totalmente cambiada, junto con los acuarios; posteriormente, día por medio se renovó las $3 / 4$ partes del agua de mar y los acuarios fueron cambiados cada tres días dependiendo del estado de ésta.

La dieta alimenticia consistió en plancton colectado en el ambiente (Kramer y Zweifel, 1970), durante los primeros 6 días posteclosión. Luego fue reemplazada por alimento artificial (Tzeng y Yu, 1992), compuesta por harina de sangre de pescado, lecitina, aceite de hígado de bacalao, harina de pescado y aceites vegetales; además, se le proporcionó nauplios de Artemia sp. como alimento vivo (Fukuhara, 1983; Barkman et al., 1981; Geffen, 1982).

Los huevos y larvas de pejerrey de mar estuvieron bajo un fotoperíodo de $14 \mathrm{hr} 15^{\prime}$ luz y $9 \mathrm{hr} 45^{\prime}$ oscuridad, que corresponde al fotoperíodo natural para los meses de diciembre y enero.

Para validar los microincrementos, el muestreo se realizó día por medio durante los primeros 17 días; desde el día 18 los muestreos se realizaron diariamente, tomándose arbitrariamente una muestra de 5 larvas al azar, fijándolas en una solución de alcohol al 80\% (Tzeng y Yu, 1992). Posteriormente, bajo lupa se les midió su longitud estándar. Los otolitos sagittae se removieron desde la cámara sacular con finas agujas de disección; luego fueron lavados con agua destilada, depositados en portaobjetos rotulados, sellados con una gota de entellán y cubiertos con un cubreobjeto (Brothers et al., 1976).

Taubert y Coble (1977) definen las líneas diarias de otolitos como un par de bandas adyacentes, una clara y una oscura. En este estudio, cada unidad compuesta por un anillo oscuro y otro claro, formadas entre el núcleo y el borde del otolito, se leyó como un incremento. Estos se contaron sin el conocimiento de la longitud, estado de desarrollo y edad de la larva, asegurando que la determinación de ésta se basa sólo en el número de incrementos de los otolitos (Brothers, 1987), contando cada portaobjetos con un número de orden independiente del día en que se obtuvo la muestra. El conteo se realizó desde el núcleo hacia la periferia del otolito, buscándose los lugares más visibles para la lectura de los microincrementos.

Las lecturas fueron realizadas 2 veces por lecto- res diferentes. En un período de dos semanas, una tercera lectura se realizó para dirimir posibles diferencias; cuando éstas fueron mayores que tres microincrementos, se descartaron. Además, se midió el radio correspondiente a la distancia mayor entre el núcleo y el borde del otolito mediante microscopio, con un aumento de 400x. Esto para determinar la relación funcional existente entre esta variable y la longitud de la larva, supuesto de los estudios de edad y crecimiento (Laroche et al., 1982). Los otolitos medidos fueron los sagittae izquierdo y derecho, verificando la existencia de posibles diferencias entre éstos mediante el uso de la prueba no paramétrica de rangos y de signos de Wilcoxon (Canavos, 1988).

\section{RESULTADOS}

La longitud estándar promedio de las larvas al eclosionar fue de 7,3 mm ( $\mathrm{n}=5$; s = 0,2), alcanzando al final del período experimental un promedio de $12,3 \mathrm{~mm}(\mathrm{n}=5 ; \mathrm{s}=0,4)$. En las larvas recién eclosionadas se observa el saco vitelino, el cual se absorbe al sexto día.

En la Fig. 1 se muestra una microfotografía de un otolito sagitta, donde se observan los microincrementos de crecimiento diario de una larva de Austromenidia regia de 23 días de edad.

La aplicación de la prueba de rangos y de signos de Wilcoxon indicó que no existen diferencias significativas en el número de microincrementos de crecimiento entre los otolitos sagittae izquierdo y derecho $(n=40 ; Z=0,426)$, y tampoco en el radio de los otolitos sagittae izquierdo y derecho ( $\mathrm{n}=40$; $\mathrm{Z}=1,229$ ). Por lo tanto, arbitrariamente se utilizó el otolito izquierdo.

La relación entre el radio del otolito $(\mathrm{RO})$ y la edad en días (t), es mejor descrita por una función polinomial de tercer grado con transformación logarítmica (Fig. 2).

$$
\log (\mathrm{RO})=3,309+0,147 \mathrm{t}-0,0007 \mathrm{t}^{2}+0,0002 \mathrm{t}^{3}
$$

La relación entre el radio del otolito y el número de microincrementos $(\mathrm{N})$, se ajusta también a una función polinomial de tercer grado (Fig. 3).

$$
\mathrm{RO}=1,744+6,405 \mathrm{~N}-0,245 \mathrm{~N}^{2}+0,005 \mathrm{~N}^{3}
$$




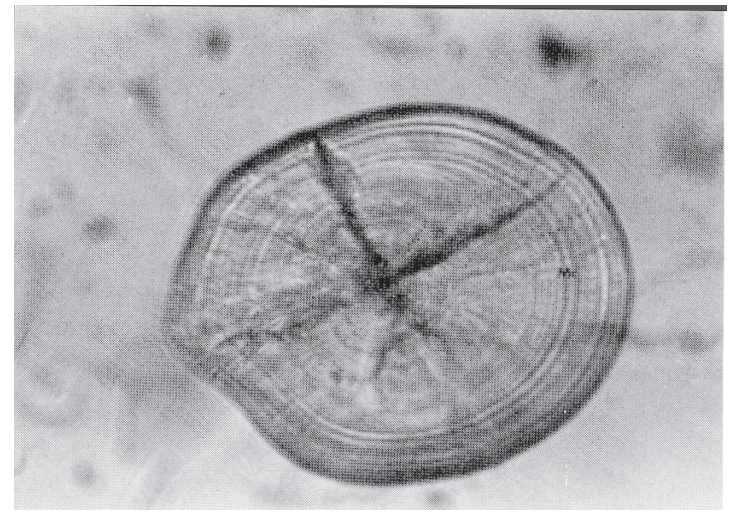

Figura 1. Microfotografía de un otolito sagittae de larva de pejerrey (Austromenidia regia) de 23 días de edad, con 29 microincrementos (aumento 400x).

Figure 1. Microphotograph of an otolite sagittae from pejerrey larvae (Austromenidia regia) 23 days age, with 29 microincrements (440x augment).

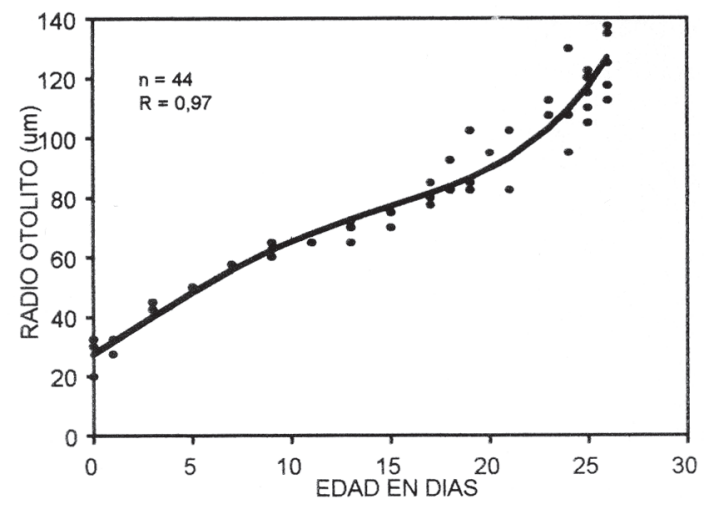

Figura 2. Ajuste de un polinomio de tercer grado entre el radio del otolito sagittae y la edad en días de larvas de pejerrey.

Figure 2. Third degree polinomial fitting for the radius of otolite sagittae as a function of age in days of pejerrey larvaes.

La regresión lineal, entre el número de microincrementos de los otolitos y la edad en días, indica que existe una estrecha relación entre estas variables (Fig. 4):

$$
\mathrm{N}=5,861+0,975 \mathrm{t} \quad\left(\mathrm{n}=54 ; \mathrm{r}^{2}=0,988\right)
$$

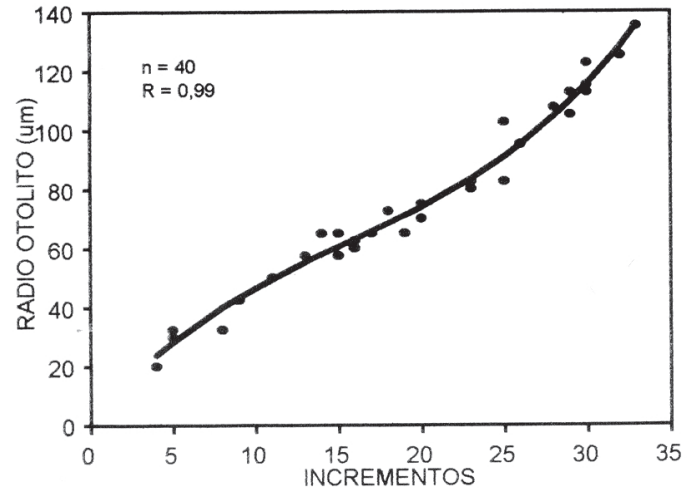

Figura 3. Ajuste del radio máximo del otolito sagittae en función del número de microincrementos en larvas de pejerrey cultivadas en laboratorio.

Figure 3. Fitting of maximun radius of otolite sagittae as a function of microincrements in pejerrey larvae.

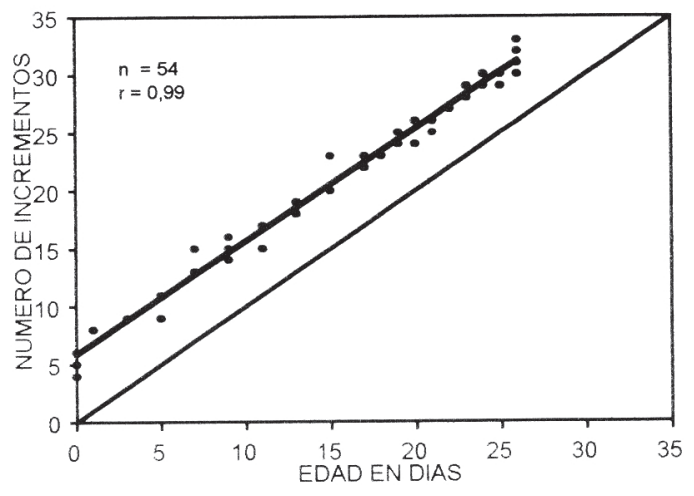

Figura 4. Microincrementos de crecimiento de otolitos sagittae en función de la edad de larvas de pejerrey cultivadas en laboratorio.

Figure 4. Growth microincrements validation in otolite saggitae as a function of time, for pejerrey larvaes.

La pendiente de la regresión (0,975 incremento/ día), no es significativamente diferente de $1\left(\mathrm{t}_{(95 \%)}=\right.$ $\left.-1,672 ; \mathrm{t}_{0,05(2) 51}=2,009\right)$. El intercepto $(5,9$ incrementos) es significativamente igual a $6\left(\mathrm{t}_{95 \%}=0,384\right)$; es decir, las larvas eclosionan con 6 microincrementos (Fig. 4), lo que se corrobora con el análisis de otolitos de larvas recién eclosionadas, los cuales presentan un promedio de 6 microincrementos $(\mathrm{n}=7 ; \mathrm{s}=0,8)$. 
El crecimiento larval de pejerrey hasta los 26 días se ajusta a un modelo polinomial de quinto grado $\left(\mathrm{R}^{2}=0,93\right)$, el cual indica que las larvas tuvieron una tasa de crecimiento negativa entre los 3 y 11 días, con un máximo de 8,5 mm de longitud estándar a los 3 días y un mínimo de $6,5 \mathrm{~mm}$ a los 11 días. Posteriormente, las larvas experimentaron una tasa de crecimiento positiva, alcanzando un promedio de 12,3 mm a los 26 días de edad (Fig. 5). El análisis de los residuos indica que no existen deficiencias en el modelo aplicado (Fig. 6).

\section{DISCUSION}

Las larvas Austromenidia regia eclosionan con una LE promedio de 7,3 mm, lo cual no es diferente a lo registrado por Chirinos y Chauman (1964) para esta especie en Perú.

Durante los primeros días de post-eclosión de las larvas el otolito sagitta es una estructura circular, con un lado cóncavo y otro convexo que va elongándose a medida que transcurre el tiempo, diferenciándose un eje antero posterior. La microestructura interna es similar a los otolitos de otras especies, conteniendo el núcleo varias primordias, lo que es característico de la familia Atherinidae (Brothers et al., 1983), observándose claramente los microincrementos de crecimiento alrededor del núcleo.

La relación entre el radio del otolito y el número de microincrementos sugiere que el crecimiento del otolito es inicialmente bajo, para posteriormente incrementar. Esto es similar al patrón general de crecimiento del otolito observado por Brothers y Mc Farland (1981) para Haemulon flavolineatum.

Otros patrones de crecimiento de otolitos han sido observados por Tzeng y Yu (1988), quienes ajustan un modelo exponencial para el patrón de los otolitos de larvas de Chanos chanos. Campana (1989) ajusta un modelo lineal para larvas de Gadus morhua, Melanogrammus aeglefinus, Pollachius virens; y Mockness y Wespestad (1992) encuentran un mejor ajuste para el crecimiento de otolitos en una función polinomial de cuarto orden en larvas de Clupea pallasii.

En la mayor parte de las especies estudiadas, los microincrementos de crecimiento en otolitos siguen un ritmo periódico de formación. En Austromenidia regia se determinó que los microincrementos de crecimiento tienen una base diaria, lo que coincide con

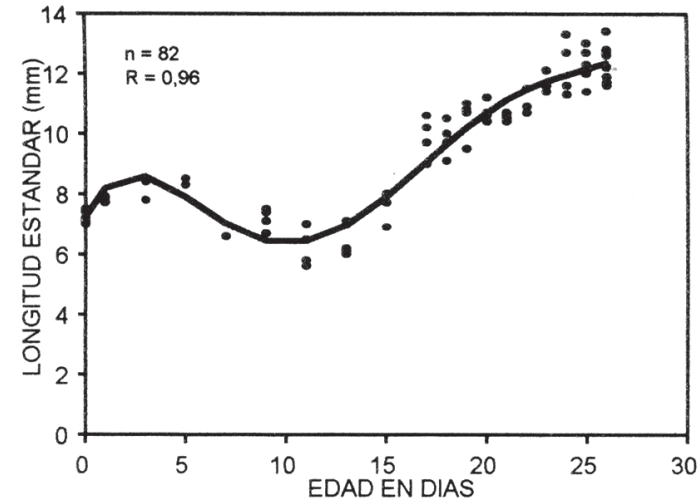

Figura 5. Crecimiento de larvas de pejerrey (Austromenidia regia) cultivadas en laboratorio por un período de 26 días.

Figure 5. Pejerrey larvaes growth raised in laboratory during 26 days.

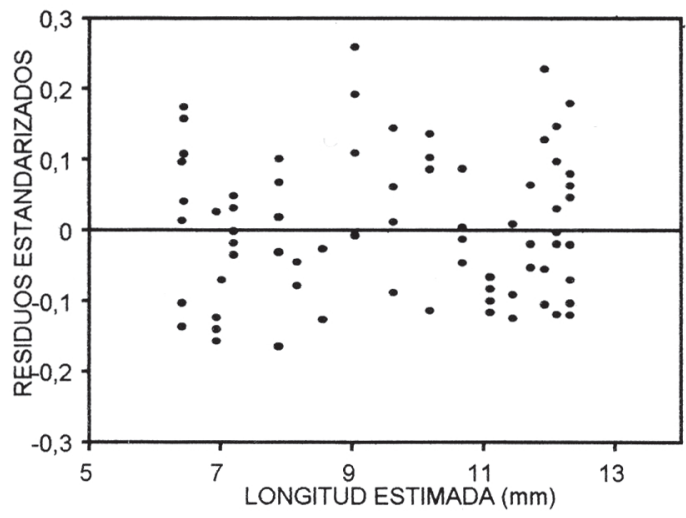

Figura 6. Gráfica de los residuos estandarizados para la relación entre la longitud estándar de la larva y la edad en días.

Figure 6. Graph of standarized residues for the relation between larvae's standard length and age in days.

los estudios realiados en los Atherínidos Leuresthes tenuis (Brothers et al., 1976) y Menidia menidia (Barkman, 1978) en condiciones de laboratorio.

Los otolitos son las primeras estructuras calcificadas que se forman durante el desarrollo de los embriones de peces. La formación de microincrementos en los otolitos antes de la eclosión parece ser un carácter de especies que tienen huevos relativamente grandes y largos períodos de 
incubación (Radtke y Dean, 1982; Brothers et al., 1976). Austromenidia regia eclosiona con 6 microincrementos, lo cual se observó a través del análisis de regresión y el examen de otolitos de larvas recién eclosionadas. Brothers et al. (1976) registran que Leuresthes tenuis eclosiona con dos microincrementos, mientras que Engraulis mordax no forma microincrementos hasta la absorción del saco vitelino o 6 días después de la eclosión (Methot y Kramer, 1979). Estas especies tienen períodos distintos de incubación, lo que indica que embriones que tienen grandes períodos de incubación y grandes sacos vitelinos pueden formar varios microincrementos antes de eclosionar; mientras que embriones que tienen períodos cortos de incubación pueden no formar microincrementos hasta que eclosionan o absorben el saco vitelino (Radtke, 1984). El hecho de saber cuándo se forma el primer microincremento de crecimiento permite conocer la edad de la larva con mayor exactitud.

Quiñónez y Gómez (1986) ajustan un polinomio de tercer grado para el crecimiento de larvas de Engraulis mordax, indicando que los modelos clásicos de ajustes (Gompertz o exponencial) pueden obscurecer eventos biológicos importantes durante la etapa larval. Moksness et al. (1993) ajustan un polinomio de quinto y tercer grado para larvas de Clupea harengus cultivadas en dos mesocosmos separados. El resultado del modelo aplicado en el presente estudio, con larvas de Austromenidia regia, indica que entre los días 3 y 11 las larvas experimentaron una tasa de crecimiento negativa; es decir, que las larvas sufrieron encogimiento corporal. Esto es factible, ya que hacia el final de la absorción del vitelo y antes de la alimentación la larva sufre un déficit de energía, lo que comienza a ser particularmente agudo cuando el vitelo desaparece completamente, y si la larva no se alimenta, comienza la autolisis (Lasker, 1965; Theilacker y Dorsey, 1980).

Jones (1984 fide Alshuth, 1988), indica que sólo bajo condiciones óptimas de cultivo los incrementos de crecimiento serían formados diariamente. Esto sugiere que los incrementos de crecimiento diario observado en larvas Austromenidia regia podrían ser el resultado de condiciones de cultivo adecuadas.

Se puede decir entonces, que la periodicidad de los microincrementos de crecimiento en larvas de pejerrey (Austromenidia regia) es diaria, formándo- se el primer microincremento de crecimiento durante la etapa de embrión.

\section{REFERENCIAS}

Alshut, S. 1988. Daily growth increments laboratory reared sprat, Sprattus sprattus L., larvae. Meeresforrch, 32: 23-29.

Barkman, R.C. 1978. The use of otolith growth rings to age young Atlantic silversides, Menidia menidia, Trans. Am. Fish. Soc., 107: 790-792.

Brothers, E.B., C.P. Mathews y R. Lasker. 1976. Daily growth increments in otoliths from larvae and adult fishes. Fish. Bull., U.S., 74(1): 1-8.

Brothers, E.B. y W.N. Mc Farland. 1981. Correlations between otolith microstructure growth, and life history transitions in newly recruited French grunt (Haemulon flavolineatum) (Desmarest) (Haemulidae). Rapp. P-v. Réun. Cons. Int. Explor. Mer., 178: 369-374.

Brothers, E.B., D.Mc. Williams y P.F. Sale. 1983. Lenght of larval life in twelve families of fishes at One Tree Lagoon, Great Barrier Reef, Australia. Mar. Biol., 76: 319-324.

Campana, S.E. 1989. Otolith microstructure of three larval gadids in the Gulf of Maine, with inferences on early life history. Can. J. Zool., 67: 1401-1410.

Campana, S.E. y J.D. Neilson. 1985. Microstructure of fish otoliths. Can. J. Fish. Aquat. Sci., 42: 10141032 .

Campana, S.E. y E. Mokness. 1991. Accuracy and precision of age and hatch date estimates from otolith microstructure examination. ICES J. Mar. Sci., 48: 303-316.

Campos, H. 1984. Los géneros de Atherínidos (Pisces: Atherinidae) del Sur de Sudamérica. Inst. Zool., Universidad Austral de Chile, pp. 71-83.

Canavos, G.C. 1988. Probabilidad y estadística: aplicaciones y métodos. McGraw-Hill/Interamericana, $651 \mathrm{pp}$.

Castillo, G., E. Aguilera, G. Herrera, P. Bernal, J. Butler, J. Chong, H. González, C. Oyarzún y C. Veloso. 1985. Larval growth rates of the pacific sardine Sardinops sagax off central Chile determi- 
nes by daily increment counts in otoliths. Biol. Pesq., 14: 11-15.

Chirinos, A. y E. Chuman. 1964. Notas sobre el desarrollo de huevos del pejerrey Odonthesthes regia regia. Biol. Inst. Mar. Perú, Callao., Vol. 1: 1-31.

Fisher, W. 1963. Die fische des brackwassergebietes lenga bei Concepción (Chile). Int. Rev. Gesamten Hydrobiol., 48: 419-511.

Fukuara, O. 1983. Development and growth of laboratory reared Engraulis japonica (Houttuyn) larvae. J. Fish. Biol., 23: 641-652.

Geffen, A.J. 1982. Otolith ring deposition in relation to growth rate in herring (Clupea harengus) and turbot (Scopthalmus maximus) larvae. Mar. Biol., 71: 317-326.

Geffen, A.J. 1983. The deposition of otolith rings in Atlantic salmon, Salmo salar L., embryos. J. Fish. Biol., 23: 467-474.

Herrera, G. y F. Balbontín. 1983. Tasa de evacuación intestinal e incidencia de alimentación en larvas de Sardinops sagax musica (Pisces: Clupeiformes). Rev. Biol. Mar., Valparaíso, 192: 113-132.

Herrera, G., E. Aguilera, G. Castillo y P. Bernal. 1985. Crecimiento de larvas de anchoveta (Engraulis ringens) en Chile Central, determinado por conteo de incrementos diarios en otolitos. Biol. Pesq., 14: 11-15.

Jones, C. y E.B. Brothers. 1987. Validation of the otolith increment aging technique for striped bass, Morone saxatilis, larvae reared under suboptimal feeding conditions. Fish. Bull., U.S., 85: 171-178.

Kramer, D. y J.R. Zweifel. 1970. Growth of anchovy larvae (Engraulis mordax Girard) in the laboratory as influenced by temperature. Calif. Coop. Oceanic Fish. Invest. Rep., 14: 84-87.

Laroche, J.L., S.L. Richardson y A.A. Rosenberg. 1982. Age and growth of a pleuronectid, Parophrys vertulus, during the pelagic larval period in Oregon coastal waters. Fish. Bull., U.S., 80: 93-104.

Lasker, R. 1965. The physiology of Pacific sardine embryos and larvae. Calif. Coop. Oceanic Fish. Invest. Rep., 10: 96-101.
Lasker, R., H.M. Feder, G.H. Theilacker y R.C. May. 1970. Feeding, growth, and survival of Engraulis mordax larvae reared in the laboratory. Mar. Biol., 5: 345-353.

Lough, R.G., M. Pennington, G.R. Bolz y A.A. Rosenberg. 1982. Age and growth of larval Atlantic herring, Clupea harengus L., in the Gulf of MaineGeorges Bank Region, based on otoliths growth increments. Fish. Bull., U.S., 80: 187-199.

Methot, R.D. (Jr.) y D. Kramer. 1979. Growth of northern anchovy Engraulis mordax, larvae in the sea. Fish. Bull., U.S., 77: 413-423.

Micheli, V. y L. Huaquín. 1985. Son útiles los otolitos para determinar la edad de las larvas en Basilichthys australis (Eigenmann?). In: Resúmenes Jornadas de Ciencias del Mar, 24-26 mayo, Coquimbo, Chile.

Moksness, E. 1992. Otolith microstructure: a new method in recruitment studies and management of herring (Clupea sp.). Dr. Philos, Thesis, Department of Fisheries and Marine Biology University of Bergen, Bergen, Norway, 106 pp.

Moksness, E. y V. Wespestad. 1992. Ageing and backcalculating growth rates of pacific herring, Clupea pallasii, larvae by reading daily otolith increments. Fish. Bull., U.S., 87: 509-513.

Moksness, E., Krukan, L. Ystanes, A. Folkvord y A. Johannessen. 1993. Comparison of somatic and otolith growth in North Sea herring (Clupea harengus L.) larvae: evaluation of growth dynamics in mesocosms. In: Recent Development in Fish Otolith Research. D.H. Sector, J.M. Dean and S.E. Campana (Eds.), pp. 119-134.

Mugiya, Y., N. Watabe y J. Yamada. 1981. Diurnal rhythm in otolith formation in the goldfish. Comp. Biochem. Phys., 68A: 659-662.

Neilson, J.D. y G.H. Geen. 1982. Otoliths of chinook salmon (Oncorhynchus tsawytscha): daily growth increments and factors influencing their production. Can. J. Fish. Aquat. Sci., 39: 1340-1347.

Neilson, J.D. y G.H. Geen. 1985. Effects of feeding regimes and diet temperature cycles on otolith increment formation juvenile chinook salmon, Oncorhynchus tsawytscha. Fish. Bull., U.S., 83: 91101.

Nishimura, A. y J. Yamada. 1984. Age of growth of larval and juvenile walleye pollock, Theragra 
chalcogramma (Pallas), as determined by otolith daily growth increments. J. Exp. Mar. Biol. Exol., 349: 191-205.

Panella, G. 1971. Fish otoliths: daily growth layers and periodical patterns. Sci., 173: 1124-1125.

Panella, G. 1980. Growth patterns in fish sagittae. In: Skeletal growth of aquatic organism. Biological records of environmental change. D. Rhoads and R. Lutz (Eds.), Planum Press. New York and London, pp. 519-560.

Quiñónez, C. y V.M. Gómez. 1986. Modelo polinomial para crecimiento individual de larvas de la anchoveta norteña, Engraulis mordax. CalCOFI Rep., Vol. XXVII, 4 pp.

Radtke, R.L. y J.M. Dean. 1982. Increment formation in the otolith of embryos, larvae and juveniles of the mummichong, Eundulus heteroclitus. Fish. Bull., U.S., 80: 201-215.

Radtke, R.L. 1984. Formation and structural composition of larval striped Mollet otoliths. Trans. Am. Fish. Sc., 143: 186-191.

SERNAP. 1994. Anuario Estadístico de Pesca. Servicio Nacional de Pesca. Ministerio de Economía, Fomento y Reconstrucción. Chile, 236 pp.

Tanaka, K., Y. Mugiya y J. Yamada. 1981. Effects of photoperiod and feeding on daily growth patterns in otoliths of juveniles Tilapia nilotica. Fish. Bull., U.S., 79: 459-466.

Taubert, B.D. y D.W. Coble. 1977. Daily rings in otolith of three species of Lepomis and Tilapia mossambica. J. Fish. Res. Board Can., 34: 332-340.
Theilacker, G.H. y K. Dorsey. 1980. Larval fish diversity, a summary of laboratory and field research. In: Workshop on the effects of environmental variation on the survival of larval pelagic fishes. IOC-UNESCO, Workshop Report, 28: 105-142.

Townsed, D.W. y J.J. Graham. 1981. Growth and age structure of larval Atlantic herring Clupea harengus harengus, in the Sheepscol river stuary Maine, as determined by daily growth increments in otoliths, Fish. Bull., U.S., 79: 123-130.

Tzeng, W.N. y S.Y. Yu. 1988. Daily growth increments in otoliths of milkfish. Chanos chanos (Forsskal), larvae. J. Fish. Biol., 32: 495-504.

Tzeng, W.N. y S.Y.Yu. 1992. Effects of starvation on the formation of daily growth increments in the otoliths of milkfish. Chanos chanos (Forsskal), larvae. J. Fish. Biol., 40:39-48.

Volk, E.C., R.C. Wissmar, C.A. Simentad y D.M. Eggers. 1984. Relationship between otolith microstructure and the growth of juvenile chum salmon (Oncorhynchus keta) under different prey rations. Can. J. Fish. Aquat. Sci., 41: 126-133.

Véliz, M. y R. Insil. 1988. Variaciones mensuales de desembarques de pejerrey (Odonthesthes regia regia) en el Perú entre 1964-1986. Biol. Inst. Mar. Perú-Callao., 1:199-302.

Wilson, K.H. y P.A. Larkin. 1980. Daily growth rings in the otoliths of juvenile sockeye salmon (Oncorhynchus nerka). Can. J. Fish. Aquat. Sci., 37: 1495-1498. 